\title{
Singularity Avoidance for Nonholonomic, Omnidirectional Wheeled Mobile Platforms with Variable Footprint
}

\author{
Alexander Dietrich, Thomas Wimböck, Alin Albu-Schäffer, and Gerd Hirzinger
}

\begin{abstract}
One characteristic attribute of mobile platforms equipped with a set of independent steering wheels is their omnidirectionality and the ability to realize complex translational and rotational trajectories. An accurate coordination of steering angle and spinning rate of each wheel is necessary for a consistent motion. Since the orientations of the wheels must align to the Instantaneous Center of Rotation (ICR), the current location and velocity of this specific point is essential for describing the state of the platform. However, singular configurations of the controlled system exist depending on the ICR, leading to unfeasible control inputs, i.e., infinite steering rates. Within this work we address and analyze this problem in general. Furthermore, we propose a solution for mobile platforms with variable footprint. An existing controller based on dynamic feedback linearization is augmented by a new potential field-based algorithm for singularity avoidance which uses the tunable leg lengths as an additional control input to minimize deviations from the nominal motion trajectory. Simulations and experimental results on the mobile platform of DLR's humanoid manipulator Justin support our approach.
\end{abstract}

\section{INTRODUCTION}

Service robotics are expected to play an important role in future domestic environments. One possibility to support a high degree of mobility is to mount manipulators on wheeled mobile platforms. Although they are restricted to move on even terrain, their flexibility and robustness are strong arguments in favor of this solution. Hence, several systems have been designed over the last decades [1], [2], [3], [4], mostly differing in the kind and number of wheels [5], [6]. A wheeled mobile platform [7] has been developed to provide the two-arm humanoid Justin [8] with a high degree of mobility, see Fig. 1. Four independent steering wheels allow to realize arbitrary linear/angular motions. Via an additional degree of freedom (dof), the leg length, each wheel may increase/decrease the distance to the central body of the platform during motion without affecting the platform height, see Fig. 2. Due to the nonholonomy of that platform system, a reorientation of the wheels is necessary to start a motion. However, the platform is omnidirectional, meaning that it can follow every planar trajectory. An example of a holonomic platform with variable footprint is given in [9].

In order to control a nonholonomic wheeled mobile robot properly and to avoid high internal forces, the wheels have to be oriented precisely at each instant. One of the most common methods is based on the explicit use/command of the Instantaneous Center of Rotation (ICR) [3], [10], [11].

The authors are with the German Aerospace Center (DLR), Institute of Robotics and Mechatronics, Wessling D-82230, Germany; Contact: alexander.dietrich@dlr.de

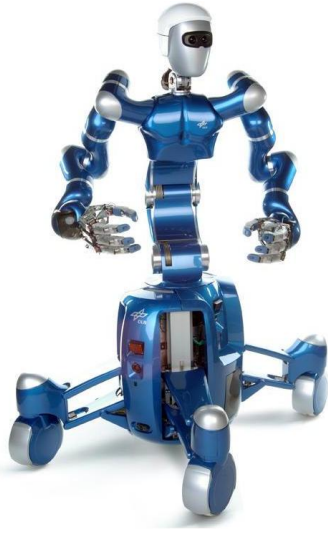

Fig. 1. DLR's humanoid Rollin' Justin.

However, singularities occur when the ICR crosses or comes close to one of the wheel axes. In [12] constraints are applied to the accessible velocity space, avoiding singular regions. Another proven method to avoid particular configurations is to consider them as obstacles and design repulsive potential fields in the respective space [13], [14]. This solution has been applied to the case of ICR-based singularities [15]. Another possibility to avoid that kind of singularity is to use special wheels [9].

The goal of this paper is to present a potential fieldbased controller for singularity avoidance in the ICR space in case of mobile platforms with variable footprint. Major contribution is the incorporation of tunable leg lengths in design and control which provide the singularity avoidance controller with an additional control input that has not yet been applied in this context before. Thereby, we are able to minimize/avoid deviations from the nominal motion trajectory which can not be achieved with a fixed platform structure. Secondly, variable instead of constant potential fields are applied in order to handle the variable footprint. Thirdly, we do not permanently use the explicit representation of the ICR due to the platform motion control via a dynamic feedback linearization. Thereby, we evade singularities which are based on the mathematical description of the ICR in contrast to existing solutions [15] wherein that issue has to be dealt with additionally.

The paper is organized as follows. After a brief introduction of the employed system, we focus on the characteristics of the ICR and the occurrence of singularities in Section III. Their effect on physical values such as wheel steering rates is addressed. In Section IV, we describe a control structure to avoid singular configurations and minimize the deviation 

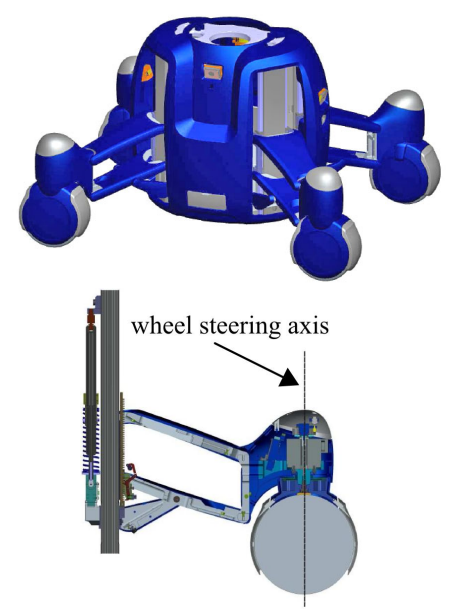
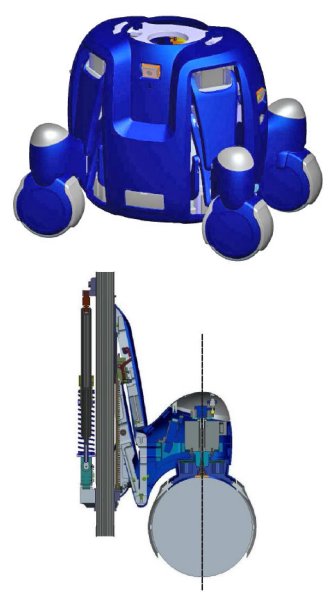

Fig. 2. The mobile platform with full extended (top left) and full retracted legs (top right) and the parallel mechanism allowing the leg extension (bottom).

from the nominal motion trajectory by incorporating the variable leg lengths. Simulations and experimental results are shown and discussed in Section V.

\section{PLATFORM OVERVIEW}

The considered mobile platform consists of a central frame connected to four centered steering wheels via a special parallel mechanism, see Fig. 2. This structure enables the wheels to extend and retract the legs independently by moving along the respective leg direction while the platform height stays unaffected [16]. For applications in narrow environments a small footprint of $812 \mathrm{~mm} \times 644 \mathrm{~mm}$ can be achieved. In case of high stability needs, e.g., for very dynamic movements or heavy load manipulations, the footprint may extend up to $1220 \mathrm{~mm} \times 1052 \mathrm{~mm}$. Absolute encoders measure steering angles and rolling velocities of the four wheels plus the related leg extensions.

Fig. 3 depicts the mobile platform schematically with $\left(x_{\mathrm{loc}}^{w}, y_{\mathrm{loc}}^{w}\right)$ representing the location of the platform center in the world frame (superscript $w$ ), $\theta$ describing the orientation, $\Phi_{i}$ and $\lambda_{i}$ with $i=1 \ldots 4$ standing for steering angle and leg extension of wheel $i$, respectively. The latter are able to move along the bold dashed lines (leg dof) w.r.t. the body frame (superscript b). A dynamic feedback linearization allows to control the translational and rotational motion as well as the leg lengths [7]. Low-level onboard controllers make it possible to realize given steering and spinning velocities of the wheels. Therefore, these values are considered as control inputs. Further details on the underlying motion control algorithm can be found in [7].

Within this paper we use body coordinates by default, hence superscript $b$ will be omitted. In case of the use of world coordinates we will refer to it explicitly.

\section{ICR AND INDUCED SINGULARITIES}

Within this work we define the Instantaneous Center of Rotation (ICR) as the unique point $\boldsymbol{z}$ in the platform body frame around which the vehicle rotates at each instant.

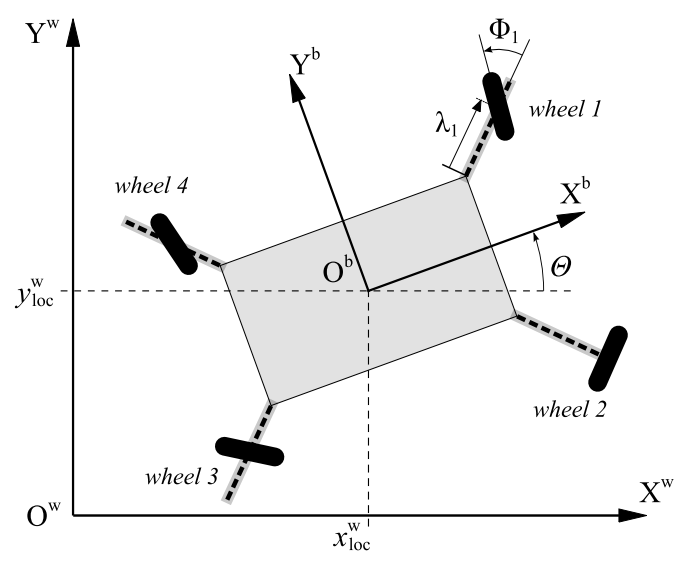

Fig. 3. Schematic plot of the mobile platform.

According to Fig. 4, the ICR in Cartesian body coordinates can be described by

$$
\begin{aligned}
& \boldsymbol{z}=\left(\begin{array}{l}
x_{\mathrm{ICR}} \\
y_{\mathrm{ICR}}
\end{array}\right)=\underbrace{\frac{v}{|\dot{\theta}|}}_{r} \cdot\left(\begin{array}{c}
\cos \gamma \\
\sin \gamma
\end{array}\right), \\
& v=\sqrt{\dot{x}^{2}+\dot{y}^{2}},
\end{aligned}
$$

where the numerator in (1) characterizes the absolute translational and the denominator the absolute angular velocity of the platform center. The whole fraction equals the radius of curvature $r$ (distance from the origin to the ICR) while $\gamma$ indicates the corresponding direction. The latter derives straightforward from the direction of motion (which is determined by $\dot{x}$ and $\dot{y}$ ) and the sense of rotation,

$$
\gamma=\arctan _{2}(\operatorname{sgn}(\dot{\theta}) \dot{x},-\operatorname{sgn}(\dot{\theta}) \dot{y}) .
$$

Concerning the order of arguments, the form $\arctan _{2}(y, x)$ is used here. The value $\mu$ in Fig. 4 specifies the direction of motion in the body frame. Obviously, an offset of $\pm \pi / 2$ to $\gamma$ exists, wherein the sign depends on the sense of rotation of the platform. Velocities $v_{w, 1}$ to $v_{w, 4}$ describe the spinning rates of the four wheels.

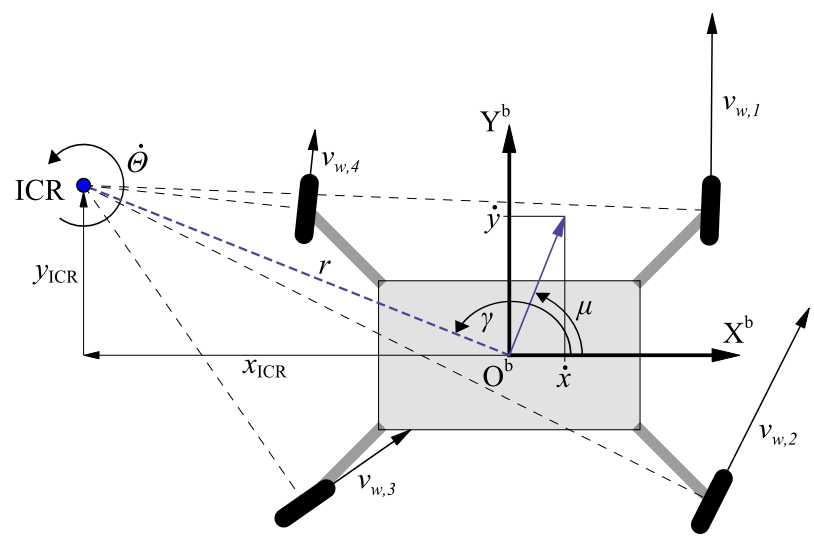

Fig. 4. Instantaneous Center of Rotation in the platform body frame (direction of motion: $\mu$; direction to the ICR: $\gamma$; rotational velocity of the platform and around the ICR: $\dot{\theta}$; wheel velocities: $v_{w, 1}$ to $v_{w, 4}$ ). 
In order to induce a consistent motion, each wheel has to orient such that its direction of motion is perpendicular to the connection line to the ICR, see Fig. 4. We would like to emphasize that this assumption only holds if a rigid body is addressed. This is not the case when the leg length $\lambda_{i}$ is varied. That leads to a separation of rigid bodies, disconnecting the former fixed contact between the respective wheel and the platform center. However, the only difference is that such a wheel obtains an additional velocity component in leg motion direction. The velocity component induced by the discussed ICR constraint remains unaffected.

It follows from the equations (1) to (3) that the transformation $(\dot{x}, \dot{y}, \dot{\theta}) \rightarrow\left(x_{\mathrm{ICR}}, y_{\mathrm{ICR}}\right)$ is not injective. This implies that several motion states of the platform lead to the same ICR, for example, doubling $v$ and $\dot{\theta}$ simultaneously does not shift $\boldsymbol{z}$. As a consequence, just setting a specific location of the ICR is not sufficient to control the whole system uniquely. An additional constraint between $\dot{x}, \dot{y}$ and $\dot{\theta}$ has to be introduced. Furthermore, it can be seen that a mathematical singularity arises when the angular velocity $\dot{\theta}$ tends towards zero, see (1). In terms of practical interpretation, this case implies that the radius of curvature tends to infinity, inducing a pure translational motion. Hence, these mathematical problems have to be dealt with when controlling a wheeled mobile robot solely via the ICR [15]. However, since we will restrict the use of the ICR representation to regions around the wheel steering axes, that kind of singularity has no relevance for our approach.

Indeed a second type of singularity might emerge during motion. We now address the necessary steering angle and rate of a wheel $i$ to fulfill the conditions of a consistent motion. No leg motion is assumed, that is $\dot{\lambda}_{i}=0 \forall i$. If the ICR passes that wheel very closely or crosses the wheel contact point, the steering rate increases rapidly to follow the desired steering angle which is forced by the ICR. Hardware limits are reached easily and a deviation from the desired steering angle causes high internal forces which stress the mechanical structure of the system.

\section{CONTROL ALGORITHM}

Although the motion controller of the Justin mobile platform, introduced in [7], is not based upon giving an explicit trajectory for the ICR, we now use its representation additionally to handle the mechanical singularities mentioned in Section III. Khatib introduced a general methodology of considering critical configurations as obstacles and modeling them as repulsive potential fields [13]. We use that concept in our approach to push the ICR away from the steering axes of the wheels. Thereby, restrictions on the nominal motion trajectory such as constraints for the radius of curvature [7] can be discarded.

\section{A. Controllability}

In [7] it is shown that one has direct access to the platform accelerations. Let us now consider their influence on the ICR velocity. Differentiating (1) w.r.t. time leads to

$$
\begin{aligned}
& \dot{\boldsymbol{z}}=\frac{\partial \boldsymbol{z}}{\partial \gamma} \dot{\gamma}+\frac{\partial \boldsymbol{z}}{\partial \dot{\theta}} \ddot{\theta}+\frac{\partial \boldsymbol{z}}{\partial v} \dot{v} \\
& =\underbrace{\left[\begin{array}{cc}
-\frac{v}{|\dot{\theta}|} \sin \gamma & -\frac{\operatorname{sgn}(\dot{\theta}) v}{\dot{\theta}^{2}} \cos \gamma \\
\frac{v}{|\dot{\theta}|} \cos \gamma & -\frac{\operatorname{sgn}(\dot{\theta}) v}{\dot{\theta}^{2}} \sin \gamma
\end{array}\right]}_{\boldsymbol{J}_{\mathrm{ICR}}}\left[\begin{array}{c}
\dot{\gamma} \\
\ddot{\theta}
\end{array}\right]+\left[\begin{array}{c}
\frac{\cos \gamma}{|\dot{\theta}|} \\
\frac{\sin \gamma}{|\dot{\theta}|}
\end{array}\right] \dot{v}
\end{aligned}
$$

for the velocity of the ICR in the plane with $\boldsymbol{J}_{\mathrm{ICR}}$ expressing a Jacobian matrix. We observe that through $\dot{\gamma}$ and $\ddot{\theta}$ we are able to control the velocity of the ICR directly as long as $\boldsymbol{J}_{\mathrm{ICR}}$ has full rank, that is $\operatorname{det} \boldsymbol{J}_{\mathrm{ICR}} \neq 0 \forall \dot{\theta} \neq 0$ :

$$
\operatorname{det} \boldsymbol{J}_{\mathrm{ICR}}=\frac{\operatorname{sgn}(\dot{\theta}) \cdot v^{2}}{|\dot{\theta}| \cdot \dot{\theta}^{2}}\left(\sin ^{2} \gamma+\cos ^{2} \gamma\right)=\frac{v^{2}}{\dot{\theta}^{3}}
$$

It results from (5) that a loss of controllability only occurs in case of a pure rotational motion $(v=0)$ or when the angular velocity tends to infinity. Both implies that the ICR is lying in the platform center. Accordingly, there is no need to control the ICR in that zone, since no singular configuration of the platform is reached.

Considering Fig. 4, a variation exclusively in $\dot{\gamma}$ makes the ICR turn around the platform (tangential) while $\ddot{\theta}$ generates a radial motion. We summarize that the two variables $\dot{\gamma}$ and $\ddot{\theta}$ have orthogonal effect on the ICR behavior which provides us with a proper control input to push away the ICR, if necessary. Furthermore, a benefit of this approach is that we can keep $\dot{v}=0$. Thereby, the second part in (4) disappears.

\section{B. Design of the Repulsive Potential Fields}

A simple approach is an axially symmetric potential field which is placed around the wheel steering axis. One choice of such a potential $V_{\text {rep }}(d)$ is

$$
V_{\text {rep }}(d)=\left\{\begin{array}{ll}
-\frac{F_{\max }}{3 d_{\text {start }}^{2}}\left(d-d_{\text {start }}\right)^{3} & \forall d \leq d_{\text {start }} \\
0 & \forall d>d_{\text {start }}
\end{array},\right.
$$

wherein $d_{\text {start }}$ is the potential field starting radius and $d$ is defined as the distance between the ICR $z$ and the nearest wheel $\boldsymbol{w}$ (in body coordinates):

$$
d=\|\boldsymbol{z}-\boldsymbol{w}\|
$$

The corresponding repulsive force is derived as follows:

$$
F_{\text {rep }}(d)=-\frac{\partial V_{\text {rep }}(d)}{\partial d}=\left\{\begin{array}{ll}
\frac{F_{\max }}{d_{\text {start }}^{2}}\left(d-d_{\text {start }}\right)^{2} & \forall d \leq d_{\text {start }} \\
0 & \forall d>d_{\text {start }}
\end{array} .\right.
$$

The force $F_{\max }$ should be set according to the maximum feasible control inputs. Fig. 5 gives an overview of the positioning of the potential fields in the body frame.

\section{Effect on the ICR}

We want to emphasize the fact that $F_{\text {rep }}(d)$, applied to the ICR, is no genuine force expressed in $[\mathrm{N}]$ at all since the ICR does not possess an inherent inertia. It has been shown in (4) that the platform accelerations have a direct impact on the ICR velocity. According to that, discontinuities in $\dot{z}$ 


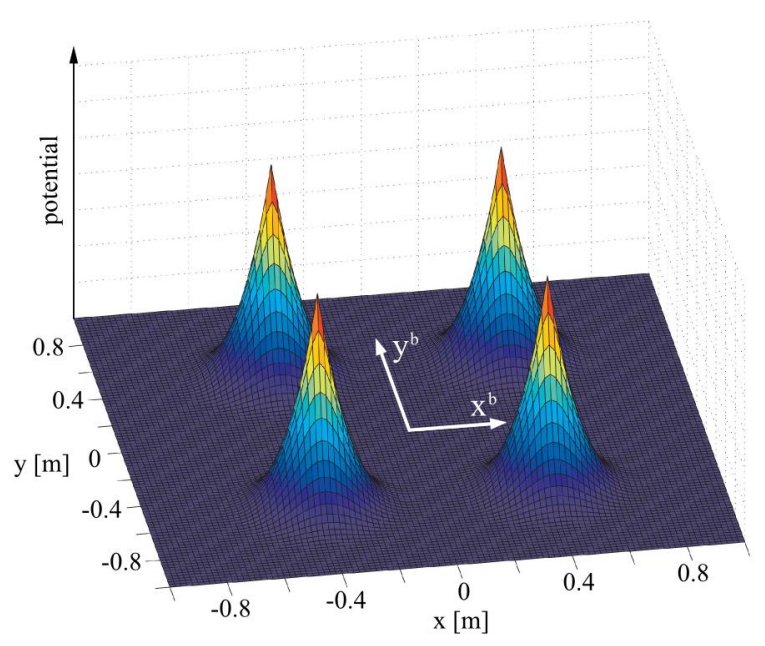

Fig. 5. Position and shape of the potentials in body frame.

are possible. However, this is beneficial as we do not have to decelerate the ICR before repelling it into the opposite direction again.

For the resulting repulsive effect on the ICR, the equation

$$
\dot{\boldsymbol{z}}_{\mathrm{des}}=F_{\mathrm{rep}}(d) \cdot \underbrace{\frac{\boldsymbol{z}-\boldsymbol{w}}{d}}_{\boldsymbol{p}}
$$

can be set up where $\dot{\boldsymbol{z}}_{\text {des }}$ is the desired ICR velocity and $\boldsymbol{p}$ expresses the normalized direction from wheel contact point to ICR. An additional damping in (9) is optional but not required since no kinetic energy is stored within the motion of the ICR.

Combining (4) and (9) and setting $\dot{z}=\dot{\boldsymbol{z}}_{\text {des }}$ leads to the required ICR controller outputs $\dot{\gamma}_{\mathrm{des}}$ and $\ddot{\theta}_{\mathrm{des}}$ :

$$
\left(\begin{array}{l}
\dot{\gamma}_{\mathrm{des}} \\
\ddot{\theta}_{\mathrm{des}}
\end{array}\right)=\boldsymbol{J}_{\mathrm{ICR}}^{-1} \cdot F_{\mathrm{rep}}(d) \cdot \boldsymbol{p} .
$$

Notice that the second part in (4) is omitted due to the constraint $\dot{v}=0$. The value $\ddot{\theta}_{\text {des }}$ can be applied by the motion controller [7], whereas $\dot{\gamma}_{\text {des }}$ has to be transformed into the corresponding accelerations $\ddot{x}_{\text {des }}$ and $\ddot{y}_{\text {des }}$. The mathematical derivation of the transformation is straightforward and based on simple geometrical considerations. Based on (3), the rotational velocity of the ICR around the platform center, see Fig. 4, is

$$
\dot{\gamma}=\frac{\partial \gamma}{\partial t}=\frac{1}{1+\left(\frac{\dot{x}}{\dot{y}}\right)^{2}} \cdot \frac{\dot{x} \ddot{y}-\dot{y} \ddot{x}}{\dot{y}^{2}} .
$$

Notice that $\operatorname{sgn}(\dot{\theta})$ from (3) has been omitted here since $\dot{\theta} \neq$ 0 in case of repulsion. Rearranging (11) with (2) delivers the instantaneous linear relation

$$
\ddot{y}=\frac{\dot{y}}{\dot{x}} \cdot \ddot{x}+\frac{v^{2}}{\dot{x}} \dot{\gamma}
$$

between $\ddot{y}$ and $\ddot{x}$ for satisfying any $\dot{\gamma}$. As our purpose is to maintain the actual translational velocity $v$, the derivative of the velocity vector and the vector itself must be perpendicular, solely allowing a vector-rotation. That condition is met by

$$
\ddot{y}=-\frac{\dot{x}}{\dot{y}} \cdot \ddot{x} .
$$

Eventually solving the linear system of equations composed of (12) and (13) leads to

$$
\left(\begin{array}{c}
\ddot{x} \\
\ddot{y}
\end{array}\right)=\dot{\gamma}\left(\begin{array}{c}
-\dot{y} \\
\dot{x}
\end{array}\right) .
$$

Finally, by replacing $\dot{\gamma}$ with the desired value $\dot{\gamma}_{\text {des }}$ we obtain

$$
\left(\begin{array}{c}
\ddot{x}_{\mathrm{des}} \\
\ddot{y}_{\mathrm{des}}
\end{array}\right)=\dot{\gamma}_{\mathrm{des}}\left(\begin{array}{c}
-\dot{y} \\
\dot{x}
\end{array}\right)
$$

to complete the control law. Notice that the applied constraint $\dot{v}=0$ is only one particular approach to deal with the noninjectivity discussed in Section III. Any other choice can be made.

\section{Repulsive Effect on the Wheel}

The leg dof makes it possible to repel the wheel $i$ from the ICR as well. In contrast to the control of the ICR, a leg motion does not result in a deviation from the nominal translational/rotational trajectory, which is the main benefit. To apply a repulsive force to the wheel, we make use of a simple mass-spring-damper equation

$$
m_{w} \ddot{\lambda}_{\mathrm{des}, i}+d_{w} \dot{\lambda}_{\mathrm{des}, i}+c_{w}\left(\lambda_{\mathrm{des}, i}-\lambda_{i, 0}\right)=F_{\mathrm{w}}
$$

where $m_{w}, d_{w}$ and $c_{w}$ describe the assigned mass of wheel $i$ and virtual values for damper and spring. $\lambda_{i, 0}$ describes the user-defined equilibrium position for the wheel location along the leg direction and $F_{\mathrm{w}}$ stands for a control input resulting from the repulsive force we want to apply. The reason for imposing the feedback gains $d_{w}$ and $c_{w}$ is the limitation of the leg length. For high efficiency around the equilibrium configuration, a non-linear increasing stiffness $c_{w}=c_{w}\left(\lambda_{\mathrm{des}, i}\right)$ is proposed as

$$
c_{w}\left(\lambda_{\mathrm{des}, i}\right)=c_{1}\left(\lambda_{\mathrm{des}, i}-\lambda_{i, 0}\right)^{2},
$$

with $c_{1}$ being a positive constant.

We propose one feasible approach for the repulsive force

$$
F_{\mathrm{w}}=-\boldsymbol{p}^{T} \boldsymbol{g} \cdot F_{\text {rep }}(d),
$$

wherein the first part describes the projection of $\boldsymbol{p}$ (from (9)) into the normalized fixed leg direction $\boldsymbol{g}$ of the nearest wheel $\boldsymbol{w}$. By inserting (17), (18) into (16) an expression for the desired leg acceleration can be developed:

$$
\ddot{\lambda}_{\mathrm{des}, i}=-\frac{\boldsymbol{p}^{T} \boldsymbol{g} F_{\mathrm{rep}}(d)}{m_{w}}-\frac{d_{w}}{m_{w}} \dot{\lambda}_{\mathrm{des}, i}-\frac{c_{1}}{m_{w}}\left(\lambda_{\mathrm{des}, i}-\lambda_{i, 0}\right)^{3} .
$$




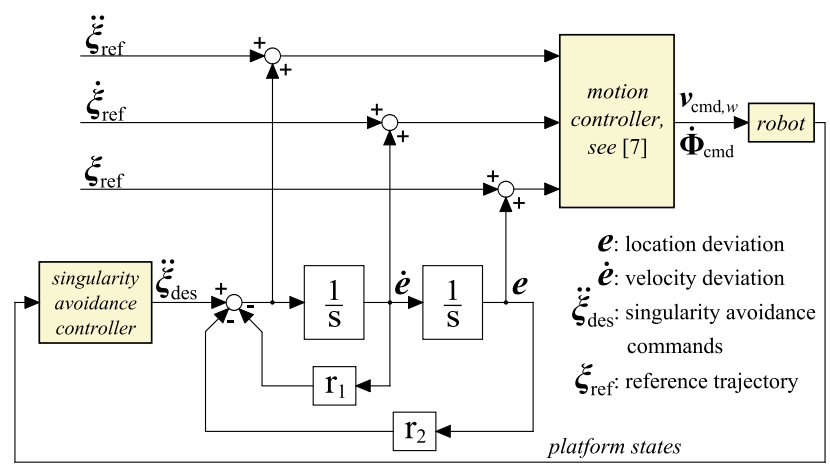

Fig. 6. Control loop to eliminate any deviations. (nominal motion trajectory

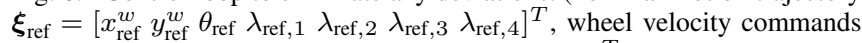
$\boldsymbol{v}_{\mathrm{cmd}, w}=\left[\begin{array}{llll}v_{\mathrm{cmd}, w, 1} & v_{\mathrm{cmd}, w, 2} & v_{\mathrm{cmd}, w, 3} & v_{\mathrm{cmd}, w, 4}\end{array}\right]^{T}$, wheel steering rate

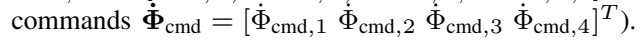

\section{E. Singularity Avoidance Controller Output}

So far, we have calculated a set of seven accelerations $\ddot{x}_{\mathrm{des}}$, $\ddot{y}_{\mathrm{des}}, \ddot{\theta}_{\mathrm{des}}$ and $\ddot{\lambda}_{\mathrm{des}, i}$ for $i=1 \ldots 4$ for singularity avoidance. We observe that a lasting deviation from the nominal trajectory of the platform would result if we just assigned these values to the motion controller, see [7]. To remedy this problem we use a separate control loop that leads back onto the nominal trajectory as soon as the ICR leaves the potential field. Fig. 6 depicts this strategy. Feedback gains $r_{1}=r_{1}(d)$ and $r_{2}=r_{2}(d)$ get activated once the potential field is left. Their values are dependent on the desired poles of that second order system. At this point it shall be mentioned that all computed accelerations $\ddot{x}_{\text {des }}, \ddot{y}_{\text {des }}, \ddot{\theta}_{\text {des }}, \ddot{\lambda}_{\text {des }, 1}, \ddot{\lambda}_{\text {des }, 2}, \ddot{\lambda}_{\text {des }, 3}, \ddot{\lambda}_{\text {des }, 4}$ have to be transformed back into the world frame before entering the control loop since the motion control algorithm [7] requires world coordinates. However, this transformation only affects $\ddot{x}_{\text {des }}$ and $\ddot{y}_{\text {des }}$. According to Fig. 6 , the singularity avoidance controller commands $\ddot{\xi}_{\text {des }}=$ $\left[\ddot{x}_{\text {des }}^{w} \ddot{y}_{\text {des }}^{w} \ddot{\theta}_{\text {des }} \ddot{\lambda}_{\text {des }, 1} \ddot{\lambda}_{\text {des }, 2} \ddot{\lambda}_{\text {des }, 3} \ddot{\lambda}_{\text {des }, 4}\right]^{T}$.

\section{F. Feasible Potential Field Expansions}

As a consequence of the possible leg motions, too widely expanded potential fields provoke overlaps if the wheels approach the platform center. This may lead to unpredictable and instable behavior of the ICR and hence of the entire platform. However, the leg's zero position $\lambda_{i, 0}$ from (16) in combination with a proper choice of $c_{w}\left(\lambda_{\operatorname{des}, i}\right)$ from (17) makes it possible to restrict the leg motion and thus the moving potential field to a certain region. A feasible approach for the expansion is depicted in Fig. 7. Depending on $\lambda_{i, 0}$ which remains within the range of the green, dotted line, the potential field center is chosen while the extension is restricted by the dashed contour in order to avoid overlaps. According to the figure, an affine relation between $d_{\text {start }, i}$ and $\lambda_{i, 0}$ exists with

$$
d_{\text {start }, i}=c_{2}+c_{3} \lambda_{i, 0} \quad \forall \lambda_{\min } \leq \lambda_{i, 0} \leq \lambda_{\max },
$$

wherein $c_{2}, c_{3} \in \mathbb{R}$. $\lambda_{\min }$ and $\lambda_{\max }$ denote the minimum and maximum leg extension, determined by the mechanical structure.

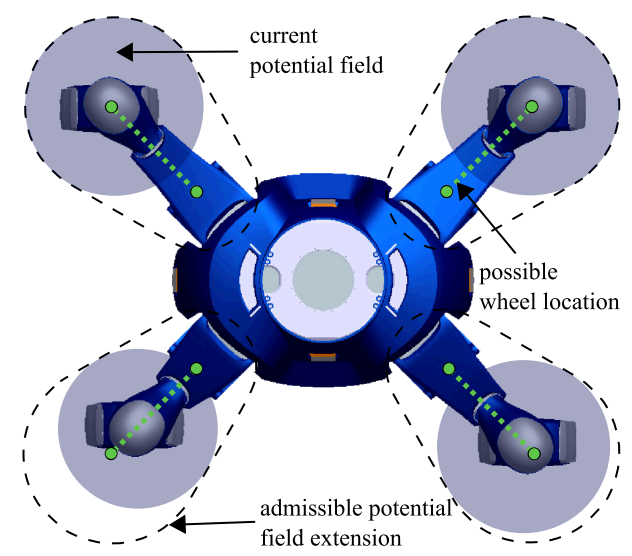

Fig. 7. Feasible potential field extensions depending on the locations of the wheels.

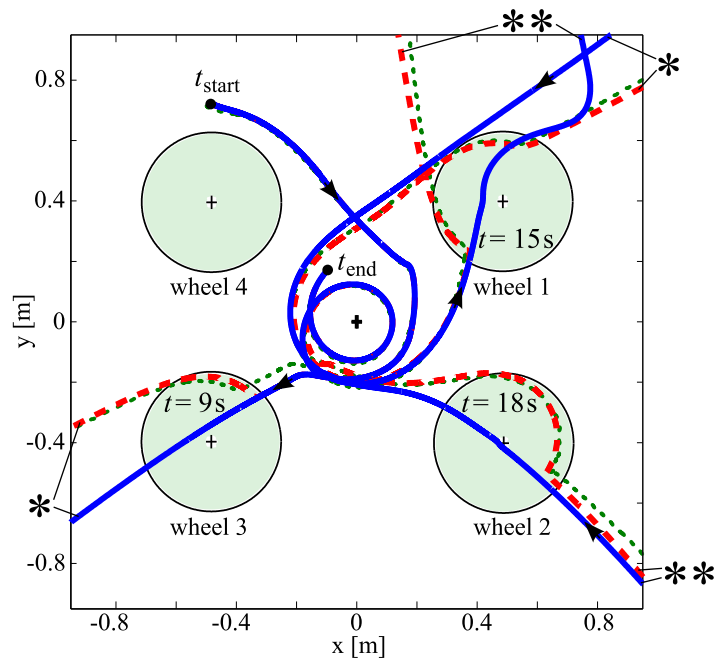

Fig. 8. ICR plot in body frame. (simulated with deactivated ICR control (blue, solid); simulated with activated ICR control (red, dashed); hardware measurements with activated ICR control (green, dotted); connections */**).

\section{RESULTS}

The control algorithm was tested for the Justin mobile platform kinematics in a Matlab/Simulink environment and implemented on the real system.

The further analysis is based on a trajectory for $x_{\mathrm{loc}}^{w}(t)$, $y_{\mathrm{loc}}^{w}(t)$ and $\theta(t)$ consisting of quintic Bézier splines. The platform achieves a rotational speed of $2.1 \mathrm{rad} / \mathrm{s}$ which requires the maximum spinning rates of the wheels, i.e., $13.9 \mathrm{rad} / \mathrm{s}$. The trajectory was implemented with and without ICR repulsion activated, while the leg lengths are initially kept constant. The platform motion with active repulsion can be seen in the video which accompanies this paper.

In Fig. 8 one can see the respective ICR behavior within the time range $\left(t_{\text {start }}, t_{\text {end }}\right)$. The potential fields (shaded circles) are crossed in case of deactivated control and avoided while the repulsion is active. Fig. 9 depicts the steering rates of wheels 1 to 3 . Whereas the steering rates stay reasonably small while the ICR control is active, they display high peaks in the non-controlled case. Critical situations occur at 9, 15 and $18 \mathrm{~s}$ which can be seen both in Fig. 8 and Fig. 9. 

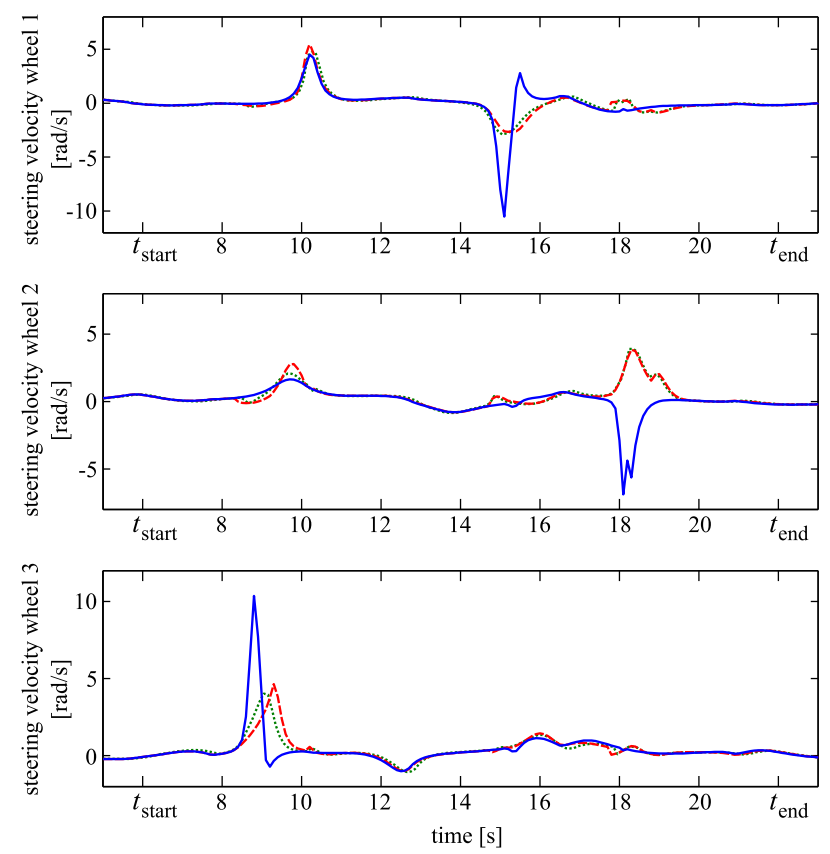

Fig. 9. Steering velocities of wheels 1,2 and 3. (simulated with deactivated ICR control (blue, solid); simulated with activated ICR control (red, dashed); hardware measurements with activated ICR control (green, dotted)).

Furthermore, we observe that the steering velocities of wheel 2 result in different signs at $18 \mathrm{~s}$. This is due to the fact that the potential field pushes the ICR around the wheel counterclockwise. Otherwise the ICR passes wheel 2 closely and clockwise as it becomes evident in Fig. 8 (non-controlled case).

At this point it shall be mentioned that the deviation induced by the ICR control may lead to a different behavior after leaving a potential field again. At $9 \mathrm{~s}$ the ICR is repelled from wheel 3 (lower left corner in Fig. 8). Before coming back onto the nominal trajectory again, the ICR approaches wheel 1 (upper right corner) while the path of the ICR does not cross the potential field if the controller is deactivated. In this exemplary case, the steering rate of wheel 1 increases slightly (at $10.5 \mathrm{~s}$ ) when the potential fields are active.

Fig. 10 shows the commanded accelerations for repelling the ICR which enter the control loop in Fig. 6. The plot at the bottom in Fig. 10 indicates whether the ICR repulsion is active (measurement). Apparently, a corrective command is applied each time the ICR leaves a potential field again. This behavior can be traced back to the feedback terms $r_{1}$ and $r_{2}$ in the control loop in Fig. 6 which are responsible for abating the location deviations. The latter stay within a range of $\pm 7 \mathrm{~cm}$ (translational) and $\pm 15 \mathrm{deg}$ (rotary) during the whole motion. These commanded errors can be reduced by using weaker potential fields. However, it still remains to be a trade-off between low steering velocity and small deviation from the desired motion trajectory.

By activating the additional leg dof, one is enabled to reduce the necessary commanded accelerations in translational and rotational direction since repelling a wheel from the ICR alleviates the need for the ICR to be pushed away. To
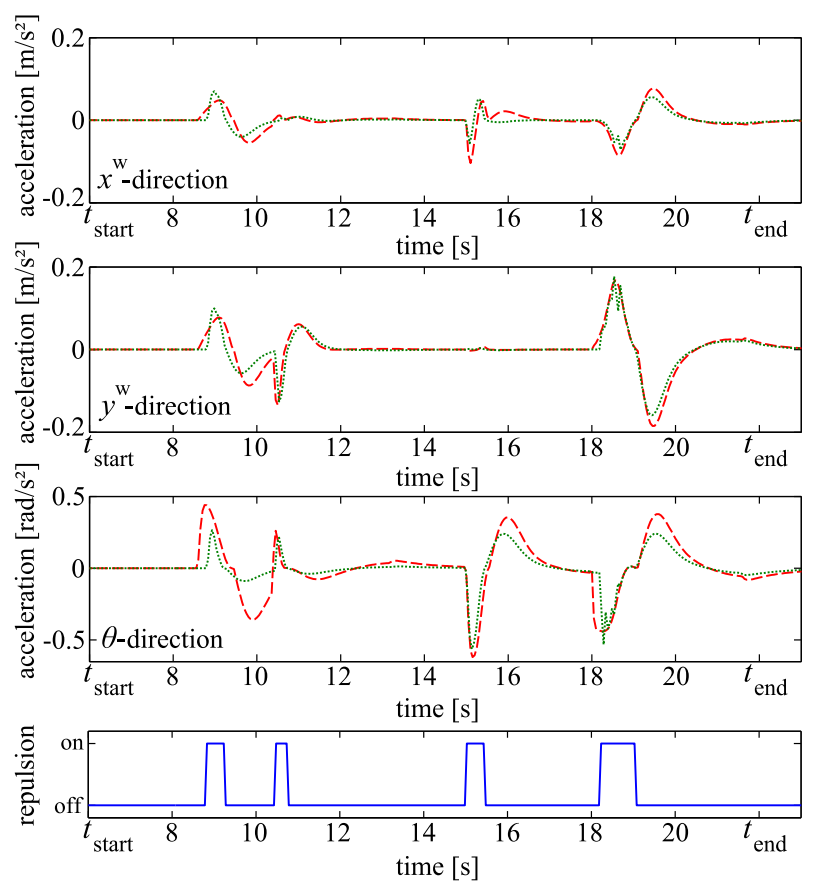

Fig. 10. Commanded accelerations in world frame and state of the ICR controller. (simulated (red, dashed); hardware measurements (green, dotted)).

support that idea, a simulated leg motion maneuver is shown in Fig. 11. Starting position for the wheel contact point is denoted A. In case of pure ICR repulsion, the wheel stays at position A. The ICR is deflected as the blue solid line demonstrates. On the contrary, the wheel moves to $\mathbf{B}$ if leg motions are enabled. The distance A-B amounts to about $5.5 \mathrm{~cm}$. Evidently, a smaller deflection from the original ICR path results (red, dashed line). That directly affects the necessary accelerations of $x_{\mathrm{loc}}^{w}, y_{\mathrm{loc}}^{w}$ and $\theta$ which are required to push away the ICR, see Fig. 12. At first, the accelerations in both cases resemble since the wheel stands still within the body frame as the ICR enters the potential field at $t_{1}$. The ICR slows down and gets stuck before it starts to pass the wheel clockwise. Meanwhile, the wheel starts to move towards $\mathbf{B}$ in case of the activated leg motion. Eventually, that reduces the effort to push away the ICR and results in the less deformed path. The leg deviation plot (down right) shows the simulated leg length deviation of wheel 2 from the equilibrium position $\lambda_{2,0}$. In this example, the reduction of the accelerations due to the activated leg motion leads to smaller maximum deviations from the reference motion trajectory. They stay 32\% (for translational motion) and 24\% (for rotational motion) below the values of locked legs.

\section{CONCLUSION AND FUTURE WORKS}

Controlling mobile platforms equipped with a set of independent steering wheels implies coping with the problem of ICR-based singularities. These configurations occur when the Instantaneous Center of Rotation passes one of the wheels' steering axes closely. In this paper, we discussed and analyzed this issue and introduced a controller which 


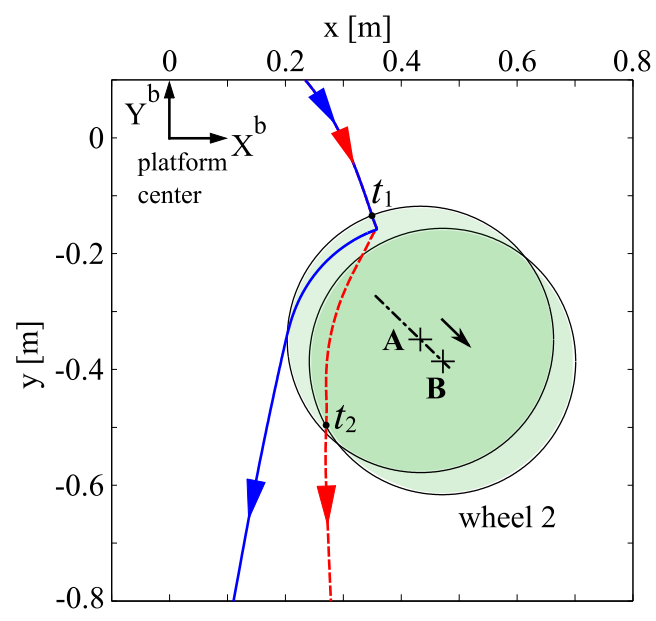

Fig. 11. Simulated ICR in body frame. (deactivated leg motion (blue, solid); activated leg motion (red, dashed); possible wheel locations (black, chain dotted)).
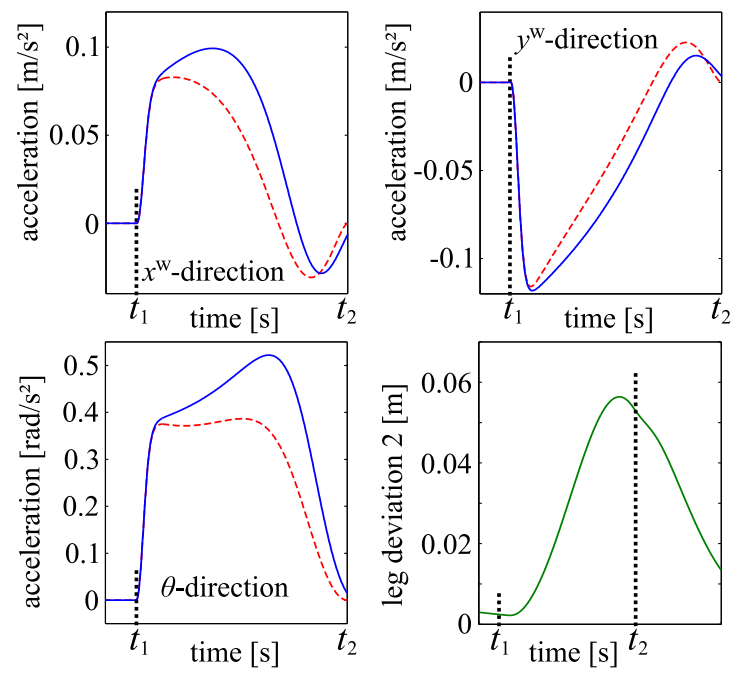

Fig. 12. Simulated acceleration commands and leg length of wheel 2 in the world frame. (accelerations for deactivated leg motion (blue, solid); accelerations for activated leg motion (red, dashed); leg deviation for activated leg motion (green, solid)).

remedies the problem. In order to avoid these singular regions, we designed repulsive potential fields around the steering axes, pushing away the ICR if it approaches a critical region. Furthermore, we introduced an additional constraint to handle the under-determined equations of the ICR control. Due to the special kinematic structure which enables variable footprint in the addressed system, namely the Justin mobile platform, we designed variable potential fields. Special attention was paid to the additional degrees of freedom which provide us with the possibility to change that footprint. An additional repulsion of the wheels from the ICR itself was achieved by incorporating the leg extension dof. The theoretical claims were validated by simulations and experimental results on the Justin mobile platform.

Future work will focus on further means to avoid the singularities and address non-reactive methods in particular. One possibility is to predict the ICR path and command the wheels so as to clear the way in advance. Another promising possibility is to use the additional velocity component due to the leg motion in order to modify the respective steering velocity systematically and avoid unfeasible peaks by distributing the steering rate onto a wider period of time. Further analyses on non-symmetric fields could also lead to an improved performance.

\section{REFERENCES}

[1] T. Asfour, K. Regenstein, P. Azad, J. Schröder, A. Bierbaum, N. Vahrenkamp, and R. Dillman, "ARMAR-III: An Integrated Humanoid Platform for Sensory-Motor Control", in Proc. of the 6th IEEERAS International Conference on Humanoid Robots, pp. 169-175, December 2006.

[2] R. Bischoff, "HERMES - A Humanoid Mobile Manipulator for Service Tasks", in Proc. of the FSR'97 Conference on Field and Service Robots, pp. 508-515, December 1997.

[3] C. Connette, A. Pott, M. Hägele, and A. Verl, "Control of an PseudoOmnidirectional, Non-holonomic, Mobile Robot based on an ICM Representation in Spherical Coordinates", in Proc. of the 47th IEEE Conference on Decision and Control, pp. 4976-4983, December 2008

[4] "Twendy-One", Sugano Laboratory, WASEDA University, online: http://www.twendyone.com, (August 2010).

[5] P. Muir and C. Neuman, "Kinematic Modeling of Wheeled Mobile Robots", Technical Report, Carnegie-Mellon University, Pittsburgh, Pennsylvania, pp. 10-13, June 1986.

[6] G. Campion, G. Bastin, and B. D'Andréa-Novel, 'Structural Properties and Classification of Kinematic and Dynamic Models of Wheeled Mobile Robots", IEEE Transactions on Robotics and Automation, Vol 12, No. 1, pp. 47-62, February 1996.

[7] P. R. Giordano, M. Fuchs, A. Albu-Schäffer, and G. Hirzinger, "On the Kinematic Modeling and Control of a Mobile Platform Equipped with Steering Wheels and Movable Legs", in Proc. of the 2009 IEEE International Conference on Robotics and Automation, pp. 4080-4087, May 2009.

[8] C. Borst, C. Ott, T. Wimböck, B. Brunner, F. Zacharias, B. Bäuml, U. Hillenbrand, S. Haddadin, A. Albu-Schäffer, and G. Hirzinger, "A humanoid upper body system for two-handed manipulation", in Proc. of the 2007 IEEE International Conference on Robotics and Automation, pp. 2766-2767, April 2007.

[9] M. Wada, and H. Asada, "Design and Control of a Variable Footprint Mechanism for Holonomic Omnidirectional Vehicles and its Application to Wheelchairs", IEEE Transactions on Robotics and Automation, vol. 15, no. 6, pp. 978-989, December 1999.

[10] M. Lauria, I. Nadeau, P. Lepage, Y. Morin, P. Giguère, F. Gagnon, D. Létourneau, and F. Michaud, "Design and Control of a Four Steered Wheeled Mobile Robot", in Proc. of the IEEE 32nd Annual Conference on Industrial Electronics, pp. 4020-4025, Nov. 2006.

[11] T. Lam, H. Qian, Y. Xu, and G. Xu, "Omni-directional Steer-by-Wire Interface for Four Wheel Independent Steering Vehicle", in Proc. of the 2009 IEEE International Conference on Robotics and Automation, pp. 1383-1388, May 2009.

[12] B. Thuilot, B. D'Andréa-Novel, and A. Micaelli, "Modeling and Feedback Control of Mobile Robots Equipped with Several Steering Wheels", IEEE Transactions on Robotics and Automation, Vol. 12, No. 3, pp. 375-390, June 1996.

[13] O. Khatib, "Real-Time Obstacle Avoidance for Manipulators and Mobile Robots", The International Journal of Robotics Research, Vol. 5, No. 1, pp. 90-98, Spring 1986.

[14] S. Shimoda, Y. Kuroda, and K. Iagnemma, "Potential Field Navigation of High Speed Unmanned Ground Vehicles on Uneven Terrain", in Proc. of the 2005 IEEE International Conference on Robotics and Automation, pp. 2828-2833, April 2005.

[15] C. Connette, C. Parlitz, M. Hägele, and A. Verl, "Singularity Avoidance for Over-Actuated, Pseudo-Omnidirectional, Wheeled Mobile Robots", in Proc. of the 2009 IEEE International Conference on Robotics and Automation, pp. 4124-4130, May 2009.

[16] M. Fuchs, Ch. Borst, P. R. Giordano, A. Baumann, E. Kraemer, J. Langwald, R. Gruber, N. Seitz, G. Plank, K. Kunze, R. Burger, F. Schmidt, T. Wimböck, and G. Hirzinger, "Rollin' Justin - Design considerations and realization of a mobile platform for a humanoid upper body", in Proc. of the 2009 IEEE International Conference on Robotics and Automation, pp. 4131-4137, May 2009. 